\title{
DETERMINING THE TIME SCALE OF A MARKOV CHAIN ${ }^{1}$
}

DAVID A. FREEDMAN

1. Introduction. A famous result of Lévy's ([3]; see also [1, pp. $121 \mathrm{ff}$.$] ) is that for a Markov chain, the transition probability from$ one state to another as a function of time $\left(p_{i k}(t)\right.$ of $\left.\S 2\right)$ is either always positive or always zero. Now the law of a given Markov chain will induce a probability in function space over the unit time interval. If the time scale of this given Markov chain is changed by a factor $\lambda$, the process remains Markovian, but with a different law. The probability induced in function space by this new law will be written $P_{\lambda}$. In view of Lévy's result, it is tempting to conjecture that all these probabilities are equivalent. This conjecture is false. Here we consider Markov chains with stable states, and show that these probabilities are equivalent precisely on the set of sample functions which are step functions. To rephrase this, if the time axis of a finite segment of sample function has been rescaled by an unknown amount, this scale factor can be calculated with probability 1 if and only if the function exhibits an infinite number of jumps.

2. The measure theory. Since we deal with many probabilities at once, it is convenient to modify the usual construction of a Markov chain. We follow [1] insofar as possible. Let $I$ be a countable set in the discrete topology, $\bar{I}=I \cup\{\infty\}$ its one-point compactification, $\delta$ any object not in $\bar{I}, \Omega$ the set of all functions $\omega$ from the non-negative rationals $R^{*}$ to $\bar{I}$, in the product topology (so that $\Omega$ is compact metrizable), $\mathcal{F}$ the $\sigma$-field of Borel subsets of $\Omega$. Let $\left\{p_{i k}(t)\right\}$ with $i, k \in I$ be a standard transition matrix function with no instantaneous states (see [1, pp. $123 \mathrm{ff} ., 149]$ ), and $\lambda$ a positive real. Then $\left\{p_{i k}(\lambda t)\right\}$ is again a standard transition matrix function with no instantaneous states. Put

$$
q_{i k}=\left.\frac{d}{d t} p_{i k}(t)\right|_{t=0} \quad \text { for } i \neq k
$$

and

$$
q_{i}=-\left.\frac{d}{d t} p_{i i}(t)\right|_{t=0} .
$$

Received by the editors October 5, 1962.

1 Prepared with the partial support of the National Science Foundation, Grant G-14648. 
It is important to remember that $\left.(d / d t) p_{i k}(\lambda t)\right|_{t=0}=\lambda q_{i k}, \quad i \neq k$; $-\left.(d / d t) p_{i i}(\lambda t)\right|_{t=0}=\lambda q_{i}$. Fix $i_{0} \in I$. Write $P_{\lambda}$ for the unique probability on $\mathcal{F}$ which makes the coordinate process $\left\{\omega(r): r \in R^{*}\right\}$ Markov with transition probabilities $\left\{p_{i k}(\lambda t)\right\}$ and $\omega(0)=i_{0}$ a.s. $\left[P_{\lambda}\right]$.

Put $\Omega_{0}=\left\{\omega \mid \omega \in \Omega ; \lim _{r \rightarrow r_{0}, r \in R^{*}} \omega(r)=\omega\left(r_{0}\right)\right.$, all $r_{0} \in R^{*} ; \lim _{r \downarrow t, r \in R^{*}} \omega(r)$

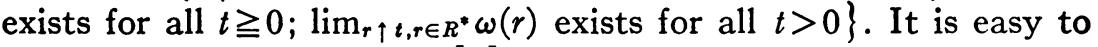
check (see Theorem 3.4 of [4]) that $\Omega_{0} \in \mathcal{F}$; and by Theorem 4 on p. 158 of [1], $P_{\lambda}\left(\Omega_{0}\right)=1$ for all $\lambda$. Define $X_{t}(\omega)=i_{0}$, for $t \geqq 0$ and $\omega \notin \Omega_{0} ;=\lim _{r \downarrow t, r \in R^{*}} \omega(r)$ for $t \geqq 0$ and $\omega \in \Omega_{0}$. The process $X .(\cdot)$ is jointly measurable, has right continuous sample functions with limits from the left; when $P_{\lambda}$ is installed on $\mathcal{F}$, it is a Markov chain with initial state $i_{0}$ and transition probabilities $\left\{p_{i k}(\lambda t)\right\}$, and the theory of $\S \mathrm{II}$ of [1] applies to it without the necessity of completing the underlying $\sigma$-fields.

The proofs in $\$ 3$ require further notation, which we introduce here. Call the inf over an empty set $+\infty, X_{\infty+0}(\omega)=\delta$, and $\infty-\infty=\infty$. Define recursively $\tau_{0}(\omega)=0, \quad \gamma_{1}(\omega)=X_{0}(\omega), \quad \tau_{n}(\omega)=\inf \left\{r \mid r \in R^{*}\right.$, $\left.r>\tau_{n-1}(\omega), X_{r}(\omega) \neq \gamma_{n}(\omega)\right\}, \gamma_{n+1}(\omega)=X_{\tau_{n}(\omega)+0}(\omega)$. Then write $\tau(\omega)$ $=\sup _{n} \tau_{n}(\omega), \sigma_{n+1}(\omega)=\tau_{n+1}(\omega)-\tau_{n}(\omega)$. Fix $D_{j}$ a sequence of finite subsets of $I$ which increase to $I$, with $D_{0}$ empty. Write $\phi_{j}(\omega)$ $=\inf \left\{r \mid r \in R^{*}, r>\tau(\omega), X_{r}(\omega) \in D_{j}\right\}, \xi_{j}(\omega)=X_{\phi_{j}(\omega)+0}(\omega)$. Let $\rho_{j}(\omega)$ $=\inf \left\{r \mid r \in R^{*}, r>\phi_{j}(\omega), X_{r}(\omega) \neq \gamma_{j}(\omega)\right\}-\phi_{j}(\omega)$. Notice that all these functions are $\mathcal{F}$-measurable. Further $\tau(\omega)$ is the least $t$, if any, such that in any neighborhood of $t, X$. ( $\omega)$ passes through an infinite number of states. If $\tau(\omega)<\infty$, and in $(0, \tau(\omega)), X$. ( $\omega)$ passes through an infinite number of states, we put $\omega \in G$. Finally, let $\mathcal{F}_{1}$ be the $\sigma$-field spanned by $\left\{X_{t} ; 0 \leqq t \leqq 1\right\}$.

3. The results. To begin with, it is clear that for all $\lambda, P_{\lambda}(\tau=1)=0$. Moreover,

THEOREM 1. The $P_{\lambda}$, restricted to $\mathcal{F}_{1}$, are equivalent on the set $\{\omega \mid \omega \in \Omega, \tau(\omega)>1\}$.

Proof. This follows by an elementary computation. Let $s$ be a finite string of symbols; the first, $s(0)$, will be a non-negative integer, and there will be $s(0)+1$ more, numbered $s(j), 1 \leqq j \leqq s(0)+1$, which are elements of $I ; s(1)=i_{0}$. Let $E(s)=\left\{\omega \mid \tau_{s(0)}(\omega)<1 \leqq \tau_{s(0)+1}(\omega)\right.$; $\left.\gamma_{j}(\omega)=s(j), 1 \leqq j \leqq s(0)+1\right\}$. Then $[\tau>1]=U_{s} E(s)$. Moreover, on $E(s), \mathcal{F}_{1}$ and the $\sigma$-field spanned by $\left\{\sigma_{j}, 1 \leqq j \leqq s(0)\right\}$ are equal. For all $P_{\lambda}$, the $\left\{\gamma_{j}: j=1, \cdots\right\}$ form a discrete time stationary Markov chain, with transition probabilities $\left\{\Pi_{i k}\right\}$, where

$$
\begin{aligned}
\pi_{i k} & =q_{i k} / q_{i} & \text { when } & i \neq k \text { and } q_{i}>0, \\
& =0 & \text { when } i=k \text { or } & q_{i}=0 .
\end{aligned}
$$


The matrix may be substochastic. See $\$ 15$ of [1]. Consequently, for each $s, P_{\lambda} E(s)$ is positive for all $\lambda$ or none, according as

$$
\left(\prod_{j=1}^{8(0)} q_{s(j)}\right) \prod_{j=1}^{8(0)} q_{s}(j) s(j+1)
$$

is positive or 0 . If this quantity is positive and $s(0)>0$ (the other cases are trivial) the conditional distribution of $\left\{\sigma_{j}, 1 \leqq j \leqq s(0)\right\}$, given $E(s)$, relative to $P_{\lambda}$ is that of the first $s(0)$ of $s(0)+1$ independent exponential random variables with parameters $\lambda q_{s(j)}$, $1 \leqq j \leqq s(0)+1$, respectively, constrained so the sum of the first $s(0)$ is less than 1 , while the sum of all is greater than or equal to 1 . As $\lambda$ varies, these distributions stay mutually equivalent, completing the proof.

TheOREM 2. The $P_{\lambda}$, restricted to $\mathcal{F}_{1}$, are mutually orthogonal on the set $\{\omega \mid \omega \in \Omega, \tau(\omega)<1\}$.

Proof. Call this set $F$. Then $F=(F \cap G) \cup(F-G)$. By Theorem 4 on p. 227 of [1], $F \cap G$ as well as $F-G$ is of positive probability simultaneously for all $P_{\lambda}$ or none. Suppose then that the first is of positive measure. Under $P_{\lambda}$, given the values of $\gamma_{j}, 1 \leqq j \leqq n+m$, in $I$, the random variables $\left\{\sigma_{j}, 1 \leqq j \leqq n\right\}$ are independent, exponential with parameters $\lambda q_{\gamma_{j}}, 1 \leqq j \leqq n$. See Theorem 2 on p. 210 of [1]. Hence, given $G$ (not $F \cap G)$, under $P_{\lambda}$ the random variables $\left\{q_{\gamma_{j}} \sigma_{j}, 1 \leqq j<\infty\right\}$ are independent exponential with common parameter $\lambda$. Thus

$$
P_{\lambda}\left\{\lim _{n \rightarrow \infty} n^{-1} \sum_{j=1}^{n} q_{\gamma_{j}} \sigma_{j}=\lambda^{-1} \mid G\right\}=1
$$

and hence

$$
P_{\lambda}\left\{(F \cap G) \text { and } \lim _{n \rightarrow \infty} n^{-1} \sum_{j=1}^{n} q_{\gamma_{j}} \sigma_{j} \neq \lambda^{-1}\right\}=0 .
$$

But the set in braces is in $\mathcal{F}_{1}$, and we may conclude that the $P_{\lambda}$, restricted to $\mathcal{F}_{1}$, are mutually orthogonal on $F \cap G$.

Next, suppose $F-G$ is of positive probability. It is not possible to use the previous argument with $\sigma_{j}$ replaced by $\rho_{j}$ because $\phi_{j+1}$ may be equal to $\phi_{j}$ with positive probability, even when $D_{j+1}$ properly contains $D_{j}$. We overcome this difficulty by a trick. Let $f_{j}(\omega)=1$ if $\phi_{j}(\omega)<\phi_{j-1}(\omega)$ and $\rho_{j}(\omega)<\infty ;=0$, elsewhere. Put $F_{n}(\omega)=\sum_{j=1}^{n} f_{j}(\omega)$, so that $F_{n}(\omega) \uparrow \infty$ on $F-G$. Now put $G_{j}(\omega)=$ least $n$ such that $F_{n}(\omega) \geqq j$, and $\hat{\rho}_{j}(\omega)=\rho_{G_{j}(\omega)}(\omega) q_{\xi_{G_{j}(\omega)}(\omega)}$. Each $\phi_{j}$ is optional in the sense of pp. $160 \mathrm{ff}$. of [1], so that by repeated applications of Theorem 2 on p. 210 of [1] the conditional distribution of $\left\{\hat{\rho}_{j}, j \geqq 1\right\}$ 
given $[\tau<\infty]-G($ not $F-G)$ relative to $P_{\lambda}$ is that of a sequence of independent exponential random variables with common parameter $\lambda$. Hence

$$
P_{\lambda}\left\{\lim _{n \rightarrow \infty} n^{-1} \sum_{j=1}^{n} \hat{\rho}_{j}=\lambda^{-1} \mid[\tau<\infty]-G\right\}=1 .
$$

But on $F-G$ let $\phi(\omega)$ be the least $j$ such that $\phi_{G_{j}(\omega)}(\omega)<1$. Since $\phi$ is finite on $F-G$, the asymptotic behavior of $n^{-1} \sum_{j=1}^{n} \hat{\rho}_{j}(\omega)$ and $n^{-1} \sum_{j=1}^{n} \hat{\rho}_{j+\phi(\omega)}(\omega)$ coincides for $\omega \in F-G$, and

$$
P_{\lambda}\left\{(F-G) \cap \lim _{n \rightarrow \infty} n^{-1} \sum_{j=1}^{n} \hat{\rho}_{j+\phi} \neq \lambda^{-1}\right\}=0 .
$$

Since the set in braces is $\digamma_{1}$-measurable, the proof terminates.

\section{REFERENCES}

1. Kai-Lai Chung, Markov chains with stationary transition probabilities, Springer, Berlin, 1960.

2. J. L. Doob, Stochastic processes, Wiley, New York, 1953.

3. P. Lévy, Processus markoviens et stationnaires. Cas dénombrable, Ann. Inst. Henri Poincaré 16 (1958), 7-25.

4. Edward Nelson, Regular probability measures on function space, Ann. of Math. (2) 69 (1959), 630-643.

University of California, Berkeley 